Cite this: RSC Advances, 2013, 3, 16060

\section{Highly sensitive biofunctionalized nickel oxide nanowires for nanobiosensing applications $†$}

\author{
Pratima R. Solanki, ${ }^{\star a b}$ Md. Azahar Ali, ${ }^{\mathrm{b}}$ Ved V. Agrawal, ${ }^{\mathrm{b}}$ A. K. Srivastava, ${ }^{\mathrm{b}}$ R. \\ K. Kotnalab and B. D. Malhotra*bc
}

Received 29th May 2013, Accepted 4th July 2013

DOI: $10.1039 / \mathrm{c} 3 \mathrm{ra42605j}$

www.rsc.org/advances

\begin{abstract}
We report results of the studies relating to the fabrication of nickel oxide nanowires (NwNiOs) with an enhanced aspect ratio of $\sim 100$ for biosensing applications. Anti Vibrio cholerae monoclonal antibodies were used to functionalize the nickel oxide nanowire $(20-80 \mathrm{~nm})$ surfaces fabricated on indium tin oxide coated glass plate for Vibrio cholerae detection. The results of the impedance response studies conducted using this immunoelectrode as a function of the Vibrio cholerae concentration revealed a detection range of 37-350 $\mathrm{ng} \mathrm{ml}^{-1}$ and a low detection limit of $0.553 \mathrm{ng} \mathrm{ml}^{-1}$ using the $3 \sigma_{\mathrm{b}} / \mathrm{m}$ criteria. The high sensitivity (11.12 $\left.\Omega\left(\mathrm{ng} \mathrm{ml}^{-1}\right)^{-1} \mathrm{~cm}^{-2}\right)$ of this fabricated sensor is attributed to the excellent electronic properties of $\mathrm{NiO}$ nanowires which facilitate the efficient transfer of electrons between the electrode and the antibody molecules through electron channeling effects. Besides this, the nanostructured NiO nanowire based immunosensor exhibits interesting supercapacitive behaviour towards the detection of CT. This immunosensor showed values of association constant $\left(K_{\mathrm{a}}\right)$ of $4.5 \times 10^{7} \mathrm{ng} \mathrm{ml}^{-1}$ and dissociation constant $\left(K_{\mathrm{d}}\right)$ of $2.22 \times 10^{-6} \mathrm{ng} \mathrm{ml}^{-1}$.
\end{abstract}

\section{Introduction}

One-dimensional (1D) metal oxide nanowires offer excellent prospects for interfacial biological recognition events with electronic signal transducers for the fabrication of miniaturized devices including biosensors, biofuel cells, nanorobotics, nanocircuits and targeted drug delivery. ${ }^{1-6}$ This is because of their small dimensional structures which can be used for the efficient transport of electrons. The high surface area and tunable electron transport properties of these interesting nanostructures arise due to quantum confinement effects; the quantum confinement effects are known to play a significant role in the operation of bionanodevices. ${ }^{7}$ In this context, functionalization of the metal oxide nanowire surface may perhaps lead to the fabrication of integrated nanowirebiomaterial hybrid systems towards the development of ultrasensitive sensors for specific recognition of desired analytes. ${ }^{8,9}$ Moreover, the biofunctionalization of metal oxide nanowires may offer an increased charge carrier density that may come close to that of the nanowire's surface. ${ }^{4}$ Wang has recently

\footnotetext{
${ }^{a}$ Special Centre for Nano Sciences, Jawaharlal Nehru university, New Delhi-110067, India. E-mail: pratimarsolanki@gmail.com; Tel: +91(11)-26704699

${ }^{b}$ Department of Science and Technology Centre for Biomedical Electronics, Biomedical Instrumentation Section, National Physical Laboratory, New Delhi110012, India

${ }^{c}$ Department of Biotechnology, Delhi Technological University, Shahbad Daulatpur, Main Bawana Road, Delhi-110042, India.E-mail: bansi.malhotra@gmail.com; Fax: 91-11-27871023; Tel: 91-11-27871043 (Ext.:1609)

$\dagger$ Electronic supplementary information (ESI) available. See DOI: 10.1039/ c3ra42605j
}

used biomolecule functionalized nanowires for the development of biosensors. ${ }^{3}$ Wan et al. have developed an immunosensor based on manganese oxide nanowires that can be used for the diagnosis and discrimination of pathogens. ${ }^{10}$

1D nickel oxide nanowires (NwNiOs) have recently been found to play an important role towards the development of nanoscale biosensing devices. ${ }^{11}$ This may be due to the electrostatic interactions between proteins and NwNiO that may perhaps facilitate biomolecule functionalization of the nanowires under physiological conditions. The presence of variable oxidation states in $\mathrm{NwNiO}$ may provide the easy mobility of electrons resulting in a fast electron transfer capability. ${ }^{12,13}$ The nanostructured NiO can be prepared using sol-gel, reverse-micelle, laser induced fragmentation and microwave irradiation techniques. ${ }^{14-17}$ The controlled growth and high aspect ratio in nanowire synthesis continue to be a challenge for the functionalization with biomolecules including antibodies. The nanostructured NiO film has recently been used for the immobilization of single-stranded DNA via electrostatic interactions for Kala-azar detection. ${ }^{13} \mathrm{~A}$ NiOchitosan based composite cholesterol biosensor via immobilization of cholesterol oxidase has recently been reported. ${ }^{14}$

The biofunctionalization of nanowires is a crucial step in the fabrication of biosensors. Non-covalent functionalization of $1 \mathrm{D}$ materials may lead to the direct attachment of functional groups $(-\mathrm{COOH},-\mathrm{OH})$ via electrostatic interactions of the biomolecules, that may preserve the structural integrity of the nanowire. ${ }^{15}$ The attachment of a single biomolecule to the nanowire surface may result in an increased electrical 
conductivity due to the edge effect. Diamond nanowires have recently been biofunctionalized using aminophenyl linker molecules to bond nucleic acid molecules for the development of a DNA biosensor. ${ }^{17}$ Niepelt et al. have reported the biofunctionalization of zinc oxide nanowires for the binding of DNA target molecules on the nanowire surface using glycidyloxypropyltrimethoxysilane as a bifunctional linker. ${ }^{18}$

Gao et al. have reported the application of antibodyfunctionalized $\mathrm{Ni}$ nanowires for cell separation. The direct attachment of antibodies onto a 1D material depends on their orientation and density. ${ }^{19,20}$ The favourable orientation of the antibodies can perhaps be achieved via binding of protein A or $\mathrm{G}$ as a linker. For example, protein $\mathrm{A}$, containing 5 homologous IgG binding domains (E, D, A, B and C), may be used to bind antibodies via non-antigenic (Fc) regions, leaving the antigen sites of the immobilized antibodies available for binding with the target antigen. ${ }^{20}$

The cholera toxin (CT) is an enterotoxin protein secreted by the bacterium Vibrio cholerae that is known to be a causative agent for diarrhea and acidosis in humans with high lethality. There is an increased demand for the availability of a rapid and sensitive analytical tool for the detection or determination of CT. Antibody or ganglioside GM1 has recently been used for the detection of CT based on different transducers. ${ }^{21,22}$ Loyprasert et al. have developed a capacitive immunosensor based on gold nanoparticles with non-conducting films of polytyramine on a gold electrode, with a detection range from $0.1 \mathrm{aM}-10 \mathrm{pM}^{22}$ A disposable amperometric immunosensor for $V$. cholerae has been fabricated using a screen-printed electrode of homemade carbon inks consisting of a mixture of polystyrene and graphite particles. In this sensor, 1-naphthyl phosphate was used as the substrate with the amperometric detection of the hydrolysis product 1-naphthol and exhibited a detection limit of $10^{5}$ cells per $\mathrm{ml}$ in $55 \mathrm{~min} .{ }^{23}$ Chiriaco et al. have developed an electrochemical impedance spectroscopy immunosensor based on mixed self-assembled monolayers (SAMs) of 11-mercaptoundecanoic acid and 2-mercaptoethanol on a gold surface. ${ }^{21}$ This electrode can be used to detect the cholera toxin from 1-100 $\mathrm{ng} \mathrm{ml} \mathrm{m}^{-1}$. The antibody of $V$. cholerae $\mathrm{O} 1$ was immobilized on a gold-coated microcantilever surface by the SAM method and the resonance frequency shift of the microcantilever was measured by dynamic force microscopy as a function of the $V$. cholerae concentration ranging from $1 \times 10^{3}$ to $1 \times 10^{7} \mathrm{CFU}$ per ml, limit of $<1 \times$ $10^{3} \mathrm{CFU}$ per $\mathrm{ml}$ and sensitivity of $<146.5 \mathrm{pg} \mathrm{Hz}{ }^{-1} \cdot{ }^{24} \mathrm{~A}$ chemiluminescence biosensor has been developed for CT detection using a supported lipid membrane as the sensing surface and a HRP-ganglioside GM1-functionalized liposome as the detection probe. ${ }^{25}$

An immunosensor based on the surface plasmon resonace (SPR) technique has been developed for the detection of $V$. cholerae using protein $\mathrm{G}$ as the linker, the free amine group of protein $\mathrm{G}$ and the carboxy groups of 11-mercaptoundecanoic acid and hexanethiol. ${ }^{26}$ A flow injection based impedimetric immunosensor has been reported for CT detection. ${ }^{20}$ Poly $(3,4-$ ethylenedioxythiophene) coated on a Nafion-supported multi- walled carbon nanotube cast film on a glassy carbon electrode has been used for the incorporation of GM1 liposomes encapsulated with an electroactive redox marker, potassium ferrocyanide, for the detection of CT and showed a linear range from $10^{-14}$ to $10^{-7} \mathrm{~g} \mathrm{ml}^{-1}$. The detection limit of this immunosensor was found to be $10^{-16} \mathrm{~g} \mathrm{ml}^{-1}$. $^{27}$

The state-of-the-art for cholera detection has resulted in the commercialization of colourimetric immunoassay testing kits like "Cholera SMART" by New Horizons Diagnostics Corporation, Columbia, Maryland, USA. Thus, there is an urgent need to develop rapid, sensitive and highly reproducible novel biosensor platforms for CT detection.

We report results of the studies relating to a monoclonal antibody-biofunctionalized NiO nanowire based ITO electrode for the development of an immunosensor for CT detection. To the best of our knowledge, this is the first time that nickel oxide nanowires have been used to develop an immunosensor for CT detection.

\section{Experimental section}

\subsection{Materials and reagents}

All chemicals have been purchased from Sigma Aldrich, USA. The monoclonal antibody specific to Vibrio cholerae (Ab-Vc), protein A (ProA) and bovine serum albumin (BSA) have been obtained from Genetix Biotech Asia Pvt. Ltd, India.

\subsection{Synthesis of $\mathrm{NiO}$ nanowires}

Nickel nitrate $\mathrm{Ni}\left(\mathrm{NO}_{3}\right)_{2} \cdot 6 \mathrm{H}_{2} \mathrm{O}$ was dissolved in deionized water to achieve a $0.5 \mathrm{M}$ solution to which a $0.2 \mathrm{M} \mathrm{KOH}$ solution was added drop wise with constant stirring at room temperature $\left(25{ }^{\circ} \mathrm{C}\right)$ until $\mathrm{pH} 9.0$ was reached. The green precipitate $\mathrm{Ni}(\mathrm{OH})_{2}$ thus obtained was centrifuged followed by washing with deionized water until neutral $\mathrm{pH}$ was reached. This solid was dried at $80^{\circ} \mathrm{C}$ overnight. A transparent viscous solution of $\mathrm{Ni}(\mathrm{OH})_{2}$ was loaded on the ITO-coated glass plate by the dipcoating technique. The $\mathrm{Ni}(\mathrm{OH})_{2}$ based films were calcinated at $400{ }^{\circ} \mathrm{C}$ for about $2 \mathrm{~h}$ to form $\mathrm{NiO}$ nanowires. The nickel hydroxide gel was prepared as shown in the reactions below.

$$
\begin{gathered}
\mathrm{Ni}\left(\mathrm{NO}_{3}\right)_{2}+2 \mathrm{KOH} \longrightarrow \mathrm{Ni}(\mathrm{OH})_{2}+2 \mathrm{~K}\left(\mathrm{NO}_{3}\right) \\
\mathrm{Ni}(\mathrm{OH})_{2} \stackrel{\Delta}{\longrightarrow} \mathrm{NwNiOs}+\mathrm{H}_{2} \mathrm{O}
\end{gathered}
$$

\subsection{Biofunctionalization of the NiO nanowire surface using antibodies via proA}

The NwNiO film surface was functionalized with monoclonal anti-Vibrio cholera (Ab-Vc) via protein A (ProA). $10 \mu \mathrm{L}$ of a ProA solution $\left(1 \mathrm{mg} \mathrm{ml}^{-1}\right)$ was uniformly spread onto the NwNiO film and incubated for about $4 \mathrm{~h}\left(25^{\circ} \mathrm{C}\right)$. During this period, electrostatic interactions occurred between the positive charges on the NwNiOs and the negative charges of ProA (Scheme 1). $10 \mu \mathrm{L}$ of a Ab- $V c$ solution $\left(1 \mathrm{ng} \mathrm{ml}^{-1}\right.$ ) was loaded onto the ProA modified NwNiO film surface. ProA facilitates the electrostatic interactions between the Fc portions of the 


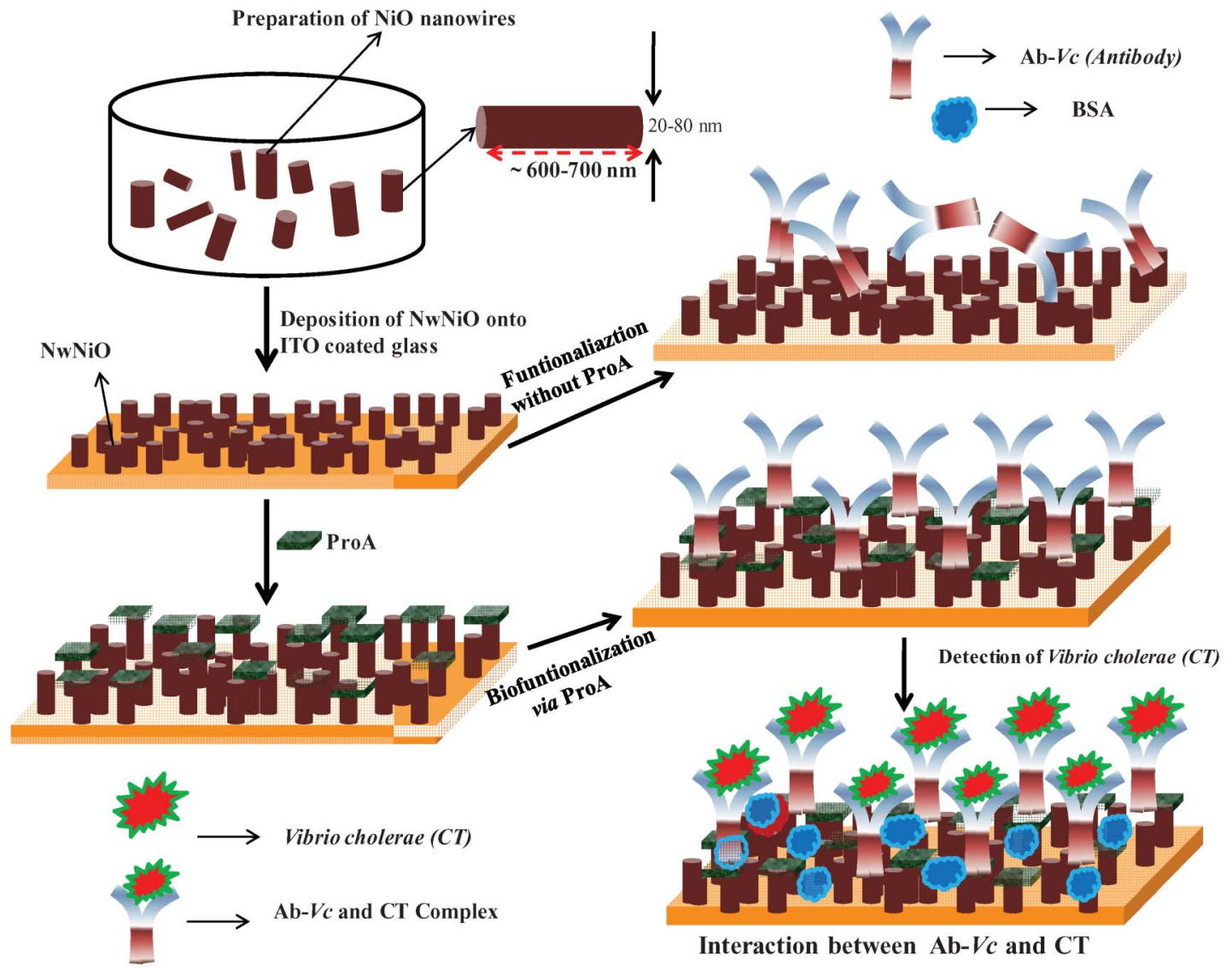

Scheme 1 The preparation of the biofunctionalized NwNiO based immunoelectrode for $\mathrm{CT}$ detection.

$\mathrm{Ab}-V c{ }^{20}$ This immunoelectrode was rinsed three times with phosphate buffer (PB; $\mathrm{pH} 7.0$ ) to remove any unbound $\mathrm{Ab}-V c$. Finally, the $\mathrm{Ab}-\mathrm{Vc} / \mathrm{NwNiO} / \mathrm{ITO}$ immunoelectrode was treated with a BSA solution (1 $\left.\mathrm{mg} \mathrm{ml} \mathrm{m}^{-1}\right)$ for about $4 \mathrm{~h}$ at room temperature $\left(25{ }^{\circ} \mathrm{C}\right)$ to block any non-specific sites. The BSA/ $\mathrm{Ab}-\mathrm{Vc} / \mathrm{NwNiO}$ based immunoelectrode was stored at $4{ }^{\circ} \mathrm{C}$ when not in use.

\subsection{Characterization}

The fabricated NwNiO/ITO, Ab-Vc/NwNiO/ITO and BSA/Ab- $V c /$ NwNiO/ITO electrodes were characterized using Fourier transform infrared spectroscopy (FT-IR, PerkinElmer, and Spectrum $\mathrm{BX}$ II). The X-ray diffraction (XRD) pattern of the NiO nanowires was obtained using a Rigaku D/Max 2200 diffractometer with $\mathrm{Cu}-\mathrm{K} \alpha$ radiation at $\lambda=1.5406 \AA$. High resolutiontransmission electron microscopy (HR-TEM, Tecnai-G2 F30 STWINT) was used to investigate the structural and morphological properties of the $\mathrm{NiO}$ nanowires and the antibodybiofunctionalized NwNiOs. Electrochemical analysis was conducted using an Autolab Potentiostat/Galvanostat (EcoChemie, AD Utrecht, The Netherlands) using a three-electrode system consisting of a working electrode, a platinum wire as the auxiliary electrode, and $\mathrm{Ag} / \mathrm{AgCl}$ as the reference electrode in phosphate buffered saline (50 mM PBS, pH 7.0) containing 5 $\mathrm{mM}\left[\mathrm{Fe}(\mathrm{CN})_{6}\right]^{3-/ 4-}$ as the redox probe. Electrochemical impedance spectroscopy (EIS) studies were conducted in the frequency range of $0.01-10^{5} \mathrm{~Hz}$, with a bias potential of $0.3 \mathrm{~V}$ and a potential period of $0.07 \mathrm{~ms}$ in $50 \mathrm{mM}$ phosphate buffered saline (PBS, containing $5 \mathrm{mM}\left[\mathrm{Fe}(\mathrm{CN})_{6}\right]^{3-/ 4-}$ ).

\section{Results and discussion}

\subsection{X-ray diffraction studies}

Fig. 1A shows the XRD pattern obtained for the NiO nanowires. The diffraction peaks at $2 \theta$ positions $37.2,43.4$, 62.9, 75.4 and 79.5 correspond to the (111), (200), (220), (311) and (222) lattice planes (JCPDS No. 78-0643), indicating a face centered cubic (FCC) structure for the NwNiOs. The obtained value for the crystallite size $(D)$ was $0.208 \mathrm{~nm}$ for the dominant peak at the (200) plane, which is in agreement with the results of HR-TEM studies.

\subsection{Fourier transform infrared spectroscopy studies}

The FT-IR spectra of NwNiOs (Fig. 1B) reveal an absorption peak at $565 \mathrm{~cm}^{-1}$ (curve a) arising from the $\mathrm{Ni}-\mathrm{O}$ vibration. The broad absorption band centered around $3578 \mathrm{~cm}^{-1}$ is attributed to the $\mathrm{O}-\mathrm{H}$ stretching vibration. It can be seen that the weak absorption bands found in the $1000-2200 \mathrm{~cm}^{-1}$ region correspond to the symmetric and asymmetric stretching vibrations of $\mathrm{O}-\mathrm{C}=\mathrm{O}$ and $\mathrm{C}-\mathrm{O}$, indicating that the $\mathrm{NwNiO}$ film perhaps facilitates a strong physical absorption of $\mathrm{H}_{2} \mathrm{O}$ 

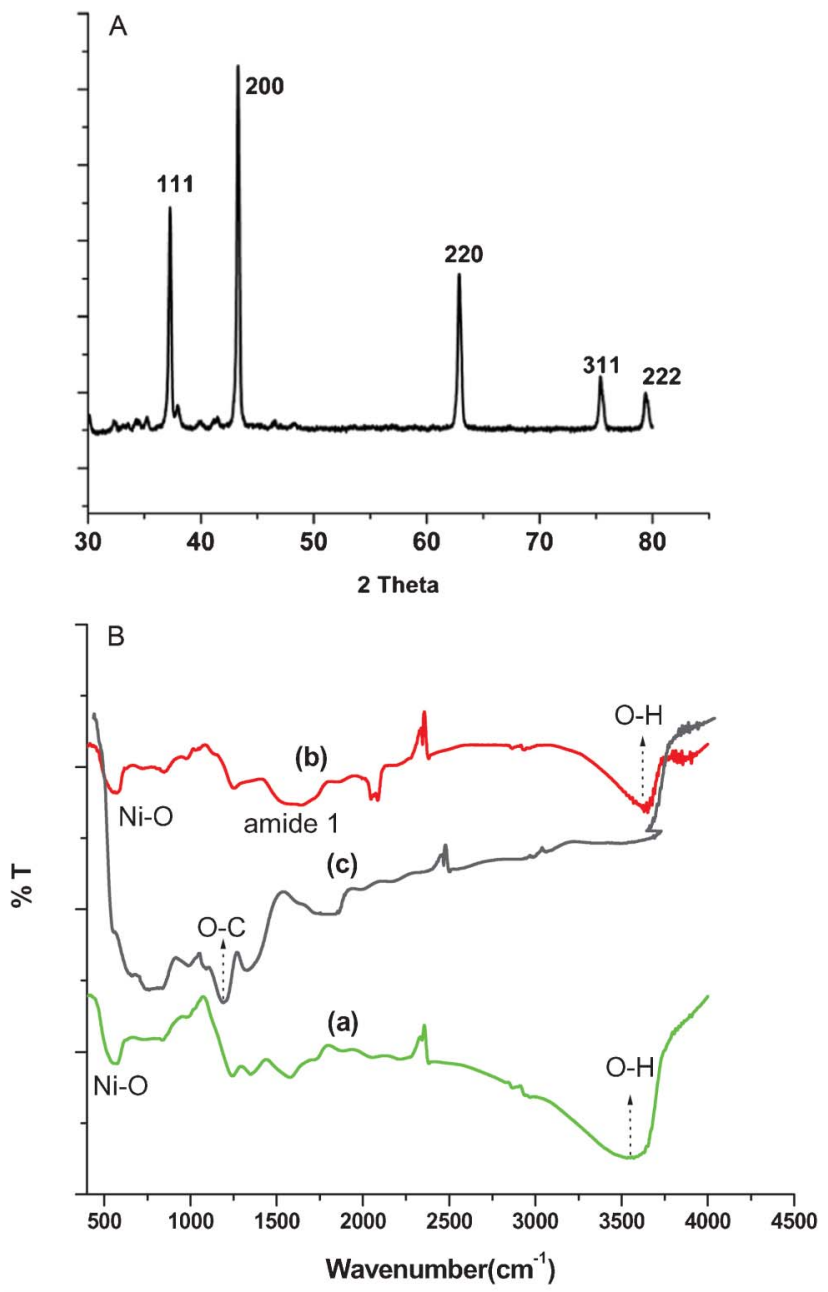

Fig. 1 (A) XRD pattern of synthesized NwNiOs. (B) FT-IR spectra of the NwNiO/ ITO electrode (a), the Ab-Vc/NwNiO/ITO immunoelectrode (b) and the BSA/Ab$\mathrm{Vc} / \mathrm{NwNiO} / \mathrm{ITO}$ immunoelectrode (c).

and $\mathrm{CO}_{2} \cdot{ }^{26}$ The presence of hydroxyl groups on the NwNiO/ITO electrode results in a hydrophilic surface that is suitable for the attachment of biomolecules. The observed broadness and shift towards lower wavenumbers of the amide 1 band (1611 $\mathrm{cm}^{-1}$ ) indicates the presence of antibodies (Ab-Vc) on the proA modified NwNiO/ITO film (curve b). The band seen at 3542 $\mathrm{cm}^{-1}$ corresponding to the $\mathrm{O}-\mathrm{H}$ stretching vibration (curve b) and the peak at about $2100 \mathrm{~cm}^{-1}$ may perhaps be due to the diimide group $(-\mathrm{N}=\mathrm{C}=\mathrm{N})$ of the antibodies. The intensity of the peak at $1195 \mathrm{~cm}^{-1}$ corresponding to $\mathrm{O}-\mathrm{C}$ is found to be enhanced due to the presence of the carboxylic groups from the antibodies (curve c). The Ni-O vibration band at $568 \mathrm{~cm}^{-1}$ becomes broader and shifts towards higher wavenumbers may be due to the incorporation of BSA. The peaks seen in the 1600-1800 $\mathrm{cm}^{-1}$ region (curve c) shift towards higher wavenumbers, indicating the presence of an amide band and revealing the immobilization of BSA onto the NwNiO/ITO film.

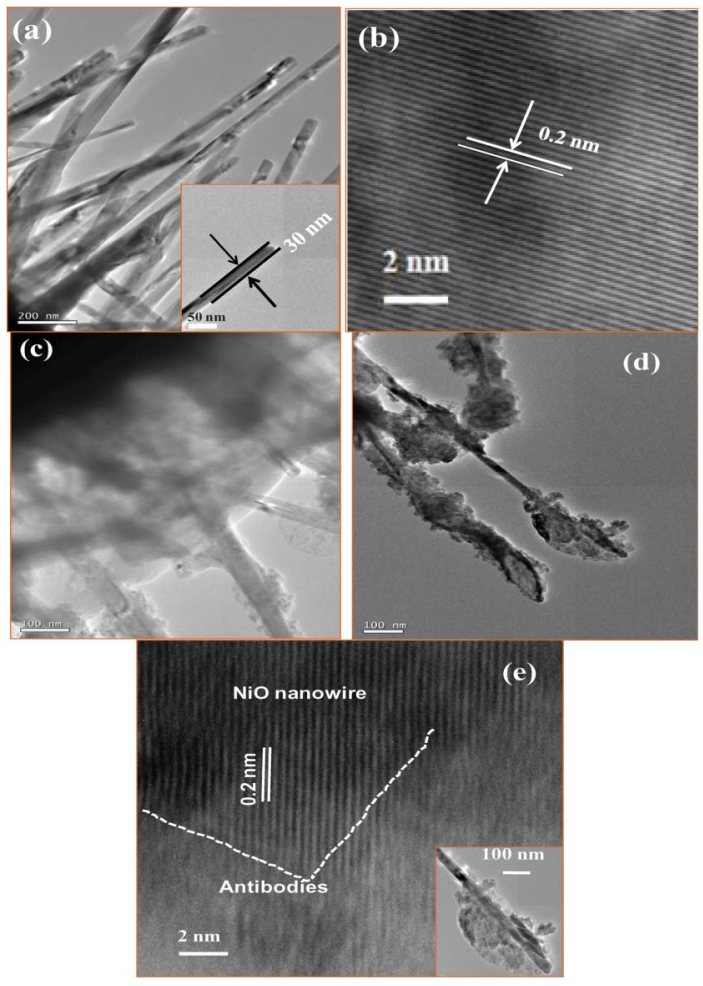

Fig. 2 HR-TEM images of (a) NiO nanowires (inset: a single nanowire), (b) lattice planes of a $\mathrm{NiO}$ nanowire, (c) antibodies immobilization on NiO nanowires, (d) individual antibodies on the nanowires and (e) a clear interface between antibodies and nanowires.

\subsection{High resolution-transmission electron microscopy (HR- TEM) studies}

HR-TEM was used to reveal the microstructure of nanostructured $\mathrm{NiO}$ and the antibodies loaded on the NiO film. The electron microscopy study of bare NiO revealed a cluster of nanowires with diameters ranging from 20 to $80 \mathrm{~nm}$. These nanowires are straight in length with an ultra thin smooth surface (Fig. 2(a)). However, the tip of these nanowires was found to be curved. The average diameter of a single nanowire was found to be about $30 \mathrm{~nm}$ (inset in Fig. 2(a)). The corresponding atomic scale image of these nanowires comprises well-organized lattice planes of NwNiOs with a free centred cubic structure (S.G. Fm $3 m, a=0.42 \mathrm{~nm}$ ). The inter plane spacing is $0.2 \mathrm{~nm}$, corresponding to the plane $h k l: 002$ (Fig. 2(b)). Fig. 2(c) shows a TEM image of the antibody (Ab-Vc) functionalized NwNiOs. Fig. 2(d) exhibits a cluster of antibodies grafted on the NiO nanowires. An individual NiO nanowire functionalized with $\mathrm{Ab}-V c$ can be clearly seen in the inset of Fig. 2(e). These individual nanowires perhaps act as carriers for $\mathrm{Ab}-\mathrm{V} c$ and hence can be utilized as probing devices. The lattice scale image (Fig. 2(e)) shows a thin layer of coating of antibodies (Ab-Vc) throughout the NwNiOs with a sharp interface between the $\mathrm{Ab}-\mathrm{Vc}$ and the atomic plane of NwNiOs at the periphery of the individual wire marked with dotted lines in Fig. 2(e). 

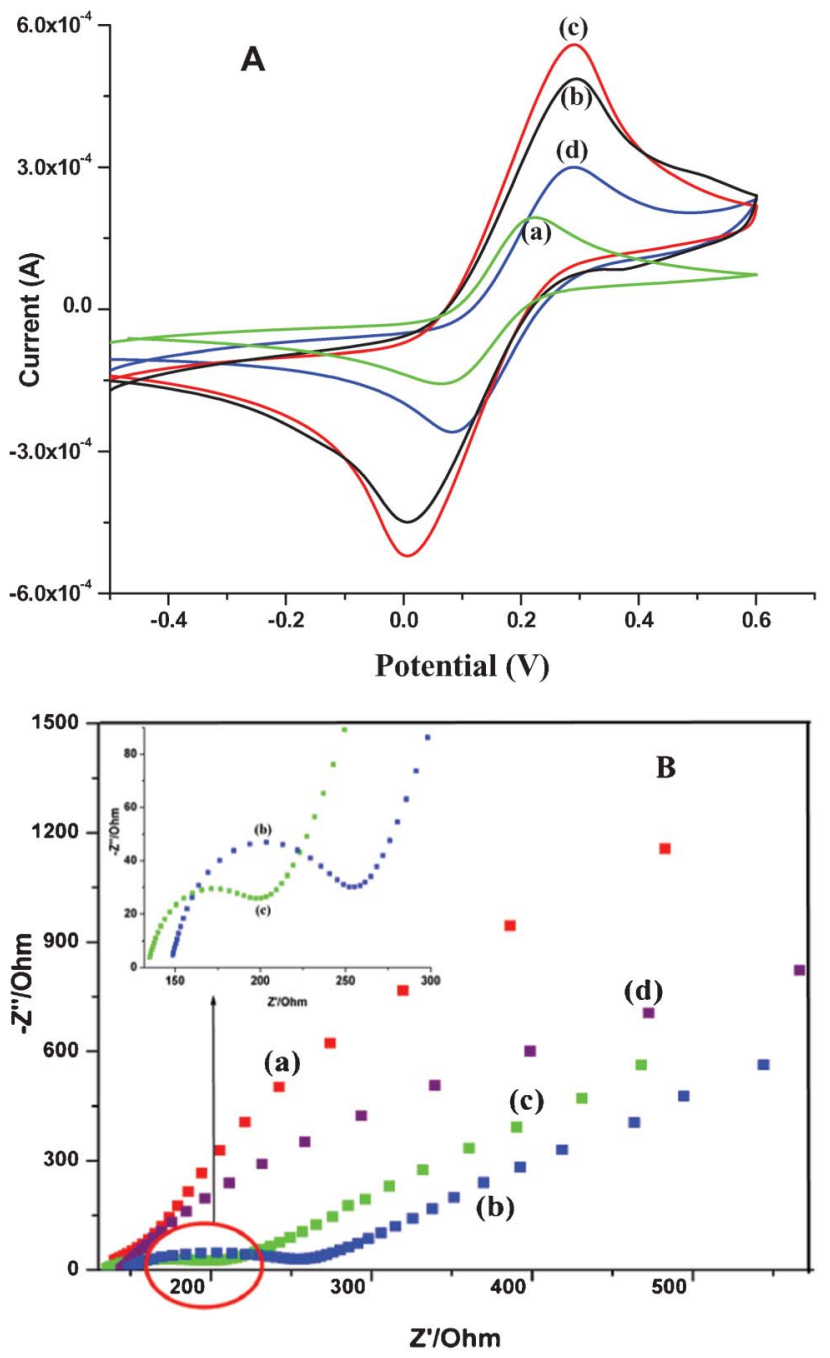

Fig. 3 (A) Cyclic voltammogram of bare ITO electrode (a), the NwNiO/ITO electrode (b), the Ab-Vc/NwNiO/ITO immunoelectrode (c) and the BSA/Ab-Vc/ $\mathrm{NwNiO} / \mathrm{ITO}$ immunoelectrode (d) at scan rates of $20 \mathrm{mV} \mathrm{s}^{-1}$. (B) EIS of the bare ITO electrode (a), the NwNiO/ITO electrode (b), the Ab- $\mathrm{Vc} / \mathrm{NwNiO} / \mathrm{ITO}$ immunoelectrode (c) and the BSA/Ab-Vc/NwNiO/ITO immunoelectrode (d).

\subsection{Cyclic voltammetry (CV) studies}

Fig. 3A shows results of the $\mathrm{CV}$ studies of the various electrodes conducted in PBS containing $5 \mathrm{mM}\left[\mathrm{Fe}(\mathrm{CN})_{6}\right]^{3-/ 4-}$ at a scan rate of $20 \mathrm{mV} \mathrm{s}^{-1}$. The magnitude of current is higher for the NwNiO/ITO electode (curve b) as compared to that of the bare ITO (curve a). This may be due to the larger apparent electrode area of the bare ITO electode. The peak-to-peak potential difference $(\Delta E)$ of the bare ITO electrode $(0.1 \mathrm{~V})$ is found to be less than that of the NwNiO/ITO electrode $(0.3 \mathrm{~V})$, due to fast electron transfer between ITO and the bulk solution. The magnitude of the current response increases after $\mathrm{Ab}-\mathrm{Vc}$ functionalizes the $\mathrm{NwNiO}$ electrode surface via ProA. The NiO nanowires act as a mediator on the electrode surface and it can perhaps significantly shorten the electron tunnelling distance between the antibodies and the electrode resulting in a higher current. Besides this, the electrostatic interaction between the free site of the antibodies $\left(-\mathrm{NH}_{2}\right.$ terminal) and the redox species may result in fast electron diffusion towards the immunoelectrode. The NiO nanowires provide a favourable environment due to their high surface-tovolume and aspect ratio resulting in the enhanced loading capacity of Ab-Vc (curve c). However, the magnitude of the current decreases with the immobilization of BSA (curve d) on the $\mathrm{Ab}-\mathrm{Vc} / \mathrm{NwNiO} / \mathrm{ITO}$ immunoelectrode surface, due to blocking of the non-binding sites on the electrode surface that may hinder the electron transfer between the medium and the electrode.

It has been found that the magnitude of both the anodic $\left(I_{\mathrm{pa}}\right)$ and the cathodic $\left(I_{\mathrm{pc}}\right)$ peak currents observed for the $\mathrm{NwNiO} / \mathrm{ITO}$ electrode and the BSA/Ab- $\mathrm{Vc} / \mathrm{NwNiO} / \mathrm{ITO}$ immunoelectrode increases linearly with the scan rate $(10-100 \mathrm{mV}$ $\mathrm{s}^{-1}$ ) (data not shown) indicating a diffusion-controlled behavior. The value of $I_{\mathrm{pc}} / I_{\mathrm{pa}}$ is greater than 1 , i.e. a quasi reversible diffusion-controlled system in both cases. The values of the slope, intercept and correlation coefficient follow eqn (1)-(4).

$$
\begin{aligned}
& I_{\mathrm{pa}}(\mathrm{A})_{\mathrm{NwNiO} / \mathrm{ITO} \text { electrode }}=4.26 \mu \mathrm{A}+7.32 \times 10^{-2} \mu \mathrm{A}\left(\frac{\mathrm{s}}{\mathrm{mV}}\right) \\
& \times \text { scan rate }\left(\frac{\mathrm{mV}}{\mathrm{s}}\right) \text { with } R^{2}=0.998 \\
& I_{\mathrm{pc}}(\mathrm{A})_{\mathrm{NwNiO} / \mathrm{ITO} \text { electrode }}=-4.35 \mu \mathrm{A}-4.32 \times 10^{-2} \mu \mathrm{A}\left(\frac{\mathrm{s}}{\mathrm{mV}}\right) \\
& \times \text { scan rate }\left(\frac{\mathrm{mV}}{\mathrm{s}}\right) \text { with } R^{2}=0.99 \\
& I_{\mathrm{pa}}(\mathrm{A})_{\mathrm{BSA} / \mathrm{Ab}-\mathrm{Vc} / \mathrm{NwNiO} / \mathrm{ITO} \text { electrode }}=1.59 \mu \mathrm{A}+1.80 \\
& \quad \times 10^{-2} \mu \mathrm{A}\left(\frac{\mathrm{s}}{\mathrm{mV}}\right) \times \text { scan rate }\left(\frac{\mathrm{mV}}{\mathrm{s}}\right) \text { with } R^{2}=0.995 \\
& I_{\mathrm{pc}}(\mathrm{A})_{\mathrm{BSA} / \mathrm{Ab}-\mathrm{Vc} / \mathrm{NwNiO} / \mathrm{TTO} \text { electrode }}=-1.31 \mu \mathrm{A}-1.02 \\
& \times 10^{-2} \mu \mathrm{A}\left(\frac{\mathrm{s}}{\mathrm{mV}}\right) \times \text { scan rate }\left(\frac{\mathrm{mV}}{\mathrm{s}}\right) \text { with } R^{2}=0.965
\end{aligned}
$$

The surface concentration of the NwNiO/ITO electrode before and after the antibody immobilization, respectively, was estimated from the plot of current versus potential (CV) using the equation: $I_{\mathrm{p}}=\frac{n^{2} F^{2} I^{*} A V}{4 R T}$ (Brown-Anson model), where $n$ is the number of electrons transferred, $F$ is the Faraday constant ( $96584 \mathrm{C} \mathrm{mol}^{-1}$ ), $I^{*}$ is the surface concentration (mol $\left.\mathrm{cm}^{-2}\right), A$ is surface area of the electrode $\left(0.25 \mathrm{~cm}^{2}\right), V$ is the scan rate $\left(20 \mathrm{mV} \mathrm{s}^{-1}\right), R$ is gas constant $\left(8.314 \mathrm{~J} \mathrm{~mol}^{-1} \mathrm{~K}^{-1}\right)$, and $T$ is the absolute temperature $(298 \mathrm{~K})$. The values of the surface concentration for the $\mathrm{NwNiO} / \mathrm{ITO}$ electrode and the $\mathrm{BSA} / \mathrm{Ab}-\mathrm{Vc} /$ $\mathrm{NwNiO} / \mathrm{ITO}$ immunoelectrode were found to be $25.7 \times 10^{-9}$ and $16.12 \times 10^{-9} \mathrm{~mol} \mathrm{~cm}^{-2}$, respectively.

\subsection{Electrochemical impedance spectroscopy (EIS) studies}

EIS provides an effective method to obtain information about the electronic features of a surface-modified electrode. Usually, the AC impedance spectra are plotted in the form of 
Nyquist diagrams i.e. $Z_{\mathrm{im}}$ as a function of $Z_{\mathrm{re}}$, with a frequency range of $0.01-10^{5} \mathrm{~Hz}$. Fig. 3B shows the Nyquist plots obtained for the bare ITO electrode (a), the NwNiO/ITO electrode (b), the $\mathrm{Ab}-V c / \mathrm{NwNiO} / \mathrm{ITO}$ immunoelectrode (c) and the BSA/Ab- $V c /$ $\mathrm{NwNiO/ITO} \mathrm{immunoelectrode} \mathrm{(d).} \mathrm{The} \mathrm{semicircle} \mathrm{diameter} \mathrm{of}$ the EIS spectra gives the value of the charge transfer resistance $\left(R_{\mathrm{CT}}\right)$ which reveals the electron transfer kinetics of the redox probe at the electrode interface. Moreover, $R_{\mathrm{CT}}$ depends on the dielectric characteristics of the electrode-electrolyte interface. It can be seen that the $R_{\mathrm{CT}}$ value $\left(1.16 \times 10^{2} \Omega\right)$ of the $\mathrm{NwNiO}$ electrode (curve b) is lower than that of the bare ITO electrode (curve a). This may perhaps be attributed to the formation of NwNiOs on the ITO surface that inhibit the direct electron flow from the redox probe $\left[\mathrm{Fe}(\mathrm{CN})_{6}\right]^{3-/ 4-}$ to the electrode surface. After the NwNiO/ITO electrode is functionalized with $\mathrm{Ab}-\mathrm{Vc}$ via ProA, the $R_{\mathrm{CT}}$ value decreases to $8.13 \times 10^{1} \Omega$ (curve c). This is due to the presence of non-binding sites in Ab-Vc that facilitate the fast electron communication between the redox probe and the immunoelectrode. In the case of the BSA/Ab- $V c / \mathrm{NwNiO} / \mathrm{ITO}$ immunoelectrode, the $R_{\mathrm{CT}}$ value is found to be $4.48 \times 10^{3} \Omega$ (curve d), revealing that the insulating properties of the BSA layer perhaps hinder the diffusion of ferricyanide ions towards the electrode.

\subsection{Electrochemical response studies}

The electrochemical impedimetric response studies of the $\mathrm{BSA} / \mathrm{Ab}-\mathrm{Vc} / \mathrm{NwNiO} / \mathrm{ITO}$ immunoelectrode were conducted as a function of the CT (Cholera toxin Vibrio cholerae) concentration (37-350 $\mathrm{ng} \mathrm{ml} \mathrm{ml}^{-1}$ ) in PBS containing $\left[\mathrm{Fe}(\mathrm{CN})_{6}\right]^{3-/ 4-}$. The $\mathrm{BSA} / \mathrm{Ab}-V c / \mathrm{NwNiO} / \mathrm{ITO}$ immunoelectrode was treated with 20 $\mu \mathrm{l}$ of CT and then incubated for five minutes $\left(25^{\circ} \mathrm{C}\right)$. It was found that the $R_{\mathrm{CT}}$ value increases with increasing concentrations of $\mathrm{CT}$ on the BSA/Ab- $V c / \mathrm{NwNiO} / \mathrm{ITO}$ immunoelectrode (Fig. 4A). The increased $R_{\mathrm{CT}}$ value indicates that the insulating layer of $\mathrm{CT}$ on the $\mathrm{BSA} / \mathrm{Ab}-V c / \mathrm{NwNiO} / \mathrm{ITO}$ immunoelectrode surface inhibits the penetration of the redox species to the electrode surface, resulting in a higher diameter of the EIS spectra and a lower charge transfer rate constant $\left(K^{0}\right){ }^{28,29}$ When the immunoelectrode was treated with a $0.2 \mathrm{M}$ glycine solution $(\mathrm{pH} 2.4)$ for about two minutes, it resulted in the regeneration of $95 \%$ of the immunoelectrode, as shown in Fig. 4A (curve g).

The plot between $R_{\mathrm{CT}}$ values and CT concentrations (37-350 ng ml${ }^{-1}$ ) yields $R_{\mathrm{CT}}(\Omega)=2913 \Omega+11.12 \Omega \mathrm{ml} \mathrm{ng}^{-1} \times \mathrm{CT}$ concentration (Fig. 4B). The sensitivity of this BSA/Ab- $V c$ / NwNiO/ITO immunoelectrode is obtained as $11.12 \Omega$ (ng $\left.\mathrm{ml}^{-1}\right)^{-1} \mathrm{~cm}^{-2}$ with a value of the linear regression coefficient $\left(R^{2}\right)$ of 0.985 as compared to those reported in the literature (Table 1). ${ }^{30,31}$ This is due to the presence of NiO nanowires which act as nanoelectrodes having the capability of fast electron transfer by establishing a conduction pathway between the electrode and the antibodies resulting in a decreased electrode impedance. It was found that the Ab-Vc functionalized $\mathrm{NiO}$ nanowire based bioelectrode exhibits improved characteristics such as a low detection limit of $0.553 \mathrm{ng} \mathrm{dl}^{-1}$ using the $3 \sigma_{\mathrm{b}} / m$ criteria, where $m$ is slope of the calibration graph and $\sigma_{\mathrm{b}}$ is the standard deviation of the blank signal, a short response time (30 s) and a good reproducibility of more than 15 times. The value of the association $\left(K_{\mathrm{a}}\right)$ and
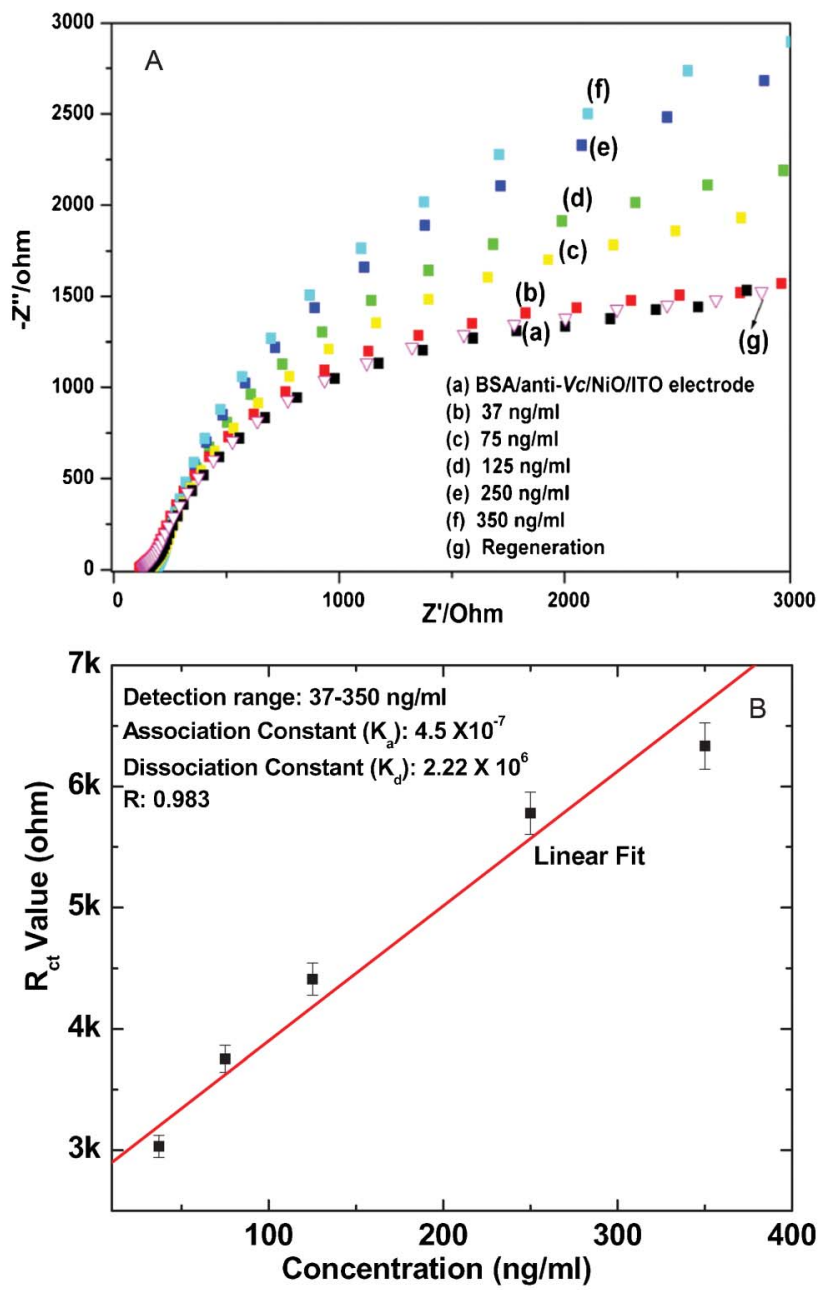

Fig. 4 (A) EIS response studies of the BSA/Ab-Vc/NwNiO/ITO immunoelectrode conducted as a function of the $\mathrm{CT}$ (Cholera toxin Vibrio cholerae) concentration (37-350 $\mathrm{ng} \mathrm{ml}^{-1}$ ) in PBS containing $\left[\mathrm{Fe}(\mathrm{CN})_{6}\right]^{3-/ 4-}$. (B) Linear plot between $R_{\mathrm{CT}}$ values vs. CT concentration (37-350 $\mathrm{ng} \mathrm{ml}^{-1}$ ).

dissociation constants $\left(K_{\mathrm{d}}\right)$ have been calculated to be $4.5 \times$ $10^{7} \mathrm{ng} \mathrm{ml}^{-1}$ and $2.22 \times 10^{-6} \mathrm{ng} \mathrm{ml}^{-1}$ respectively, indicating a strong specific affinity towards CT. The capacitive response of the $\mathrm{BSA} / \mathrm{Ab}-\mathrm{Vc} / \mathrm{NwNiO} / \mathrm{ITO}$ immunoelectrode has been described in the supplementary section. It was observed that the capacitive response decreases with increasing CT concentrations (ESI $\dagger$ data, Fig. A).

\subsection{Specificity test of the BSA/Ab-Vc/NwNiO/ITO immunoelectrode}

The specificity of the BSA/Ab- $V c / \mathrm{NwNiO} / \mathrm{ITO}$ immunoelectrode was determined using other water borne pathogens including Escherichia coli and Salmonella typhi. The BSA/Ab-Vc/NwNiO/ ITO immunoelectrode was treated with $20 \mu \mathrm{l}$ of these pathogens and the electrochemical response was measured after completion of the incubation time $(5 \mathrm{~min}$ ) (data not shown). During these measurements, it was observed that there was no significant increase in the $R_{\mathrm{CT}}$ values after interaction of the pathogens with the BSA/Ab-Vc/NwNiO/ITO 


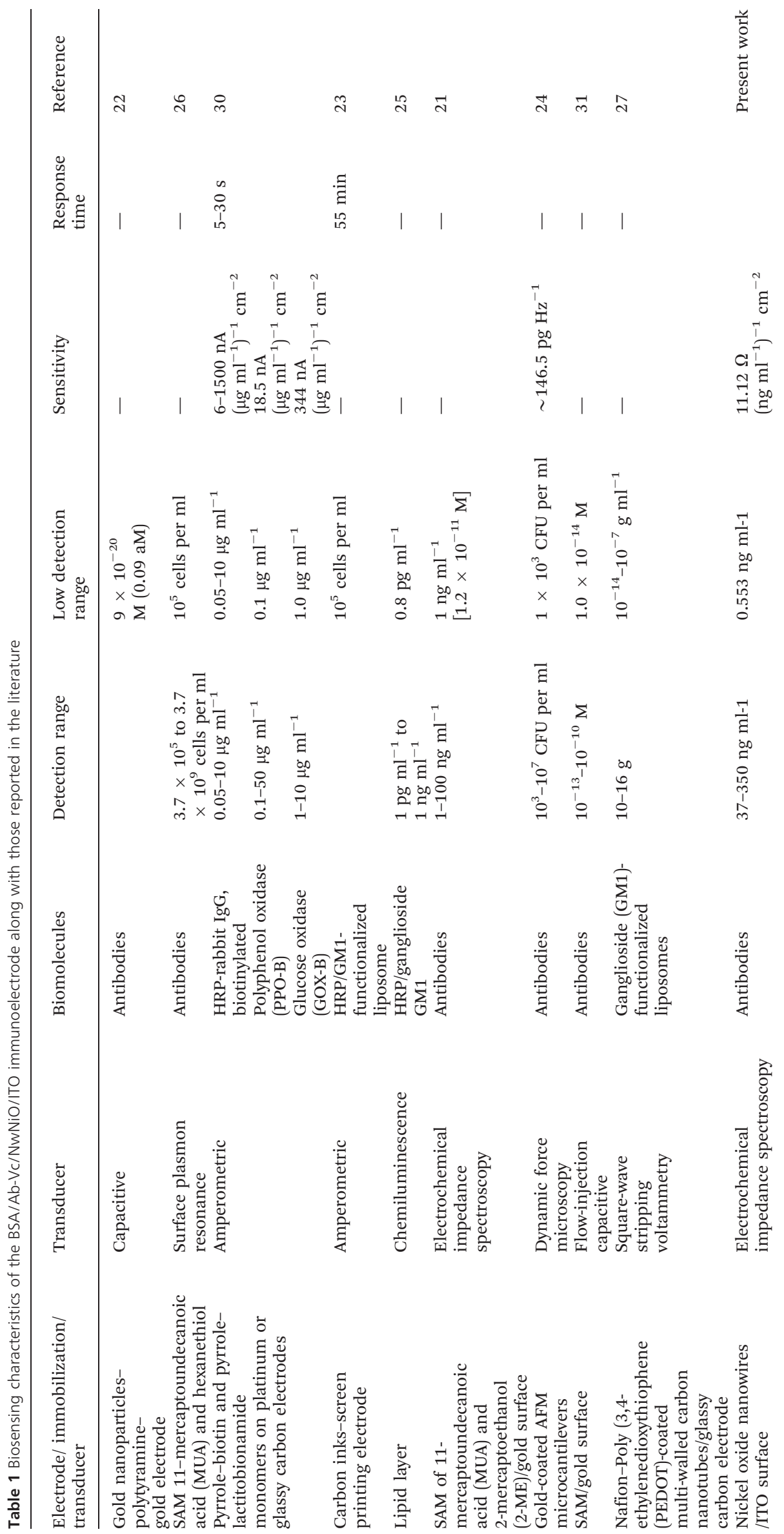


immunoelectrode, thus indicating specificity of the probe for Vibrio cholerae.

The storage stability of the BSA/Ab- $V c / \mathrm{NwNiO} / \mathrm{ITO}$ immunoelectrode was determined by observing the $R_{\mathrm{CT}}$ value at regular intervals of time for 90 days (ESI $\dagger$ data, Fig. B). The $\mathrm{BSA} / \mathrm{Ab}-\mathrm{Vc} / \mathrm{NwNiO} / \mathrm{ITO}$ immunoelectrode was stored at $4{ }^{\circ} \mathrm{C}$ when not in use. It was found that the bioelectrode exhibited a $90 \%$ response for up to 40 days. After that, the $R_{\mathrm{CT}}$ value decreased reaching $80 \%$ after 90 days.

\section{Conclusions}

In summary, a novel impedimetric immunosensor based on antibody biofunctionalized $\mathrm{NiO}$ nanowires for the sensitive electrochemical detection of Vibrio cholerae has been fabricated. The HR-TEM images showed individual nanowires that serve as carriers for the anti-Vibrio cholerae antibodies (Ab-Vc) via protein A (ProA) and bovine serum albumin (BSA). These NiO nanowires provide a favourable environment for the immobilization of $\mathrm{Ab}-V c$ due to the similarity in size with the $\mathrm{Ab}-V c$ molecules and the high surface-to-volume and aspect ratios resulting in an enhanced loading capacity of the Ab-Vc. This immunosensor based on antibody-biofunctionalized NwNiO offers an excellent detection range, a low detection limit $\left(0.553 \mathrm{ng} \mathrm{m} \mathrm{ml}^{-1}\right)$, fast response $(30 \mathrm{~s})$, high sensitivity $\left(11.12 \Omega\left(\mathrm{ng} \mathrm{ml}^{-1}\right)^{-1} \mathrm{~cm}^{-2}\right)$ and good regeneration capacity (95\%). Further efforts should be made to utilize the NiO nanowires for the sensitive detection of other environmental and clinically important pathogens such as E. coli, Salmonella typhi, etc.

\section{Acknowledgements}

We thank Dr R.C.Budhani, Director, National Physical Laboratory, New Delhi, India for the facilities. PRS is grateful to the Department of Science and Technology (DST), India for financial support. Md. Azahar Ali thanks the CSIR for a Senior Reserch Fellowship, CSIR. We thank members of the Biomedical Instrumentation Section, NPL for interesting discussions.

\section{References}

1 Y. Hu, C. Hoh, D. J. Reilly, J. Xiang, C. M. Lieber and C. M. Marcus, Nat. Nanotechnol., 2007, 2, 622.

2 J. P. Liu, C. X. Guo, C. M. Li, Y. Y. Li, Q. B. Chi, X. T. Huang, L. Liao and T. Yu, Electrochem. Commun., 2009, 11, 202.

3 J. Wang, ChemPhysChem, 2009, 10, 1748.

4 L. D. Vico, M. H. Sorensen, L. Iversen, D. M. Rogers, B. S. Sorensen, M. Brandbyge, J. Nygrd, K. L. Martinez and J. H. Jensen, Nanoscale, 2011, 3, 706.

5 N. S. Ramgir, Y. Yang and M. Zacharias, Small, 2010, 6, 1705.
6 M. A. Bangar, D. J. Shirale, W. Chen, N. V. Myung and A. Mulchandani, Anal. Chem., 2009, 81, 2168.

7 Z. P. Wei, M. Arredondo, H. Y. Peng, Z. Zhang, D. L. Guo, G. Z. Xing, Y. F. Li, L. M. Wong, S. J. Wang, N. Valanoor and T. Wu, ACS Nano, 2010, 4, 4785.

8 G. Shen, Recent Pat. Nanotechnol., 2008, 2, 160.

9 R. Wang, C. Ruan, D. Kanayeva, K. Lassiter and Y. Li, Nano Lett., 2008, 8, 2625.

10 Y. Wan, P. Qi, D. Zhang, J. Wu and Y. Wang, Biosens. Bioelectron., 2012, 33, 69.

11 G. Shen, P. C. Chen, K. Ryu and C. Zhou, J. Mater. Chem., 2009, 19, 828.

12 P. R. Solanki, A. Kaushik, V. V. Agrawal and B. D. Malhotra, NPG Asia Mater., 2011, 3, 17.

13 S. Mohan, P. Srivastava, S. N. Maheshwari, S. Sundar and R. Prakash, Analyst, 2011, 136, 2845.

14 J. Singh, P. Kalita, M. K. Singh and B. D. Malhotra, Appl. Phys. Lett., 2011, 98, 123702.

15 B. M. N. Tahir, F. Natalio, H. A. Therese, A. Yella, N. Metz, M. R. Shah, E. Mugnaioli, R. Berger, P. Theato, H. C. Schroder, W. E. G. Muller and W. Tremel, Adv. Funct. Mater., 2009, 19, 285.

16 M. K. Singh, A. Agarwal, R. Gopal, R. K. Swarnkar and R. K. Kotnala, J. Mater. Chem., 2011, 21, 11074.

17 B. Z. Yang, Z. Xie, H. Liu, F. Yan and H. Ju, Adv. Funct. Mater., 2008, 18, 3991.

18 R. Niepelt, U. C. Schroder, J. Sommerfeld, I. Slowik, B. Rudolph, R. Moller, B. Seise, A. Csaki, W. Fritzsche and C. Ronning, Nanoscale Res. Lett., 2011, 6, 511.

19 N. Gao, H. Wang and E. H. Yang, Nanotechnology, 2010, 21, 105.

20 G. P. Anderson, M. A. Jacoby, F. S. Ligler and K. D. King, J. Phys. Chem. B, 2008, 112, 6708.

21 M. S. Chiriaco, E. Primiceri, E. D. Amone, R. E. Ionescu, R. Rinaldi and M. G. Lab, Lab Chip, 2011, 11, 658.

22 S. Loyprasert, M. Hedstrom, P. Thavarungkul, P. Kanatharana and B. Mattiasson, Biosens. Bioelectron., 2010, 25, 1977.

23 V. K. Rao, M. K. Sharma, A. K. Goel, L. Singh and K. Sekhar, Anal. Sci., 2006, 22, 1207.

24 U. Sungkanak, A. Sappat, A. Wisitsoraat, C. Promptmas and A. Tuantranont, Biosens. Bioelectron., 2010, 26, 784.

25 H. Chen, Y. Zheng, J. H. Jiang, H. L. Wu, G. L. Shen and R. Q. Yu, Biosens. Bioelectron., 2008, 24, 684.

26 J. Y. Jyoung, S. Hong, W. Lee and J. W. Choi, Biosens. Bioelectron., 2006, 21, 2315.

27 S. Viswanathan, L. Wu, M. R. Huang and J. A. Ho, Anal. Chem., 2006, 78, 1115-1121.

28 P. R. Solanki, A. Kaushik, T. Manaka, M. K. Pandey, M. Iwamoto, V. V. Agrawal and B. D. Malhotra, Nanoscale, 2010, 2, 2811.

29 H. Qiao, Z. Wei, H. Yang, L. Zhu and X. Yan, J. Nanomater., 2009 Article ID 795928, 1.

30 R. E. Ionescub, C. Gondranc, S. Cosnierc, L. A. Ghebera and R. S. Marksa, Talanta, 2005, 66, 15.

31 M. Labib, M. Hedstrom, M. Amin and B. Mattiasson, Anal. Chim. Acta, 2009, 634, 255. 